\title{
SULLA DERIVATA SECONDA MISTA DI UN INTEGRALE DOPPIO.
}

\author{
Nota di Guido Fubini (Torino) e Leonida Tonelli (Parma).
}

\section{Adunanza del 13 febbraio 1916.}

I.

(da una lettera di Leonida Toneli a Guido Fubini).

\section{Egregio Professore,}

Desidererei sottoporle la seguente questione. Consideriamo la funzione

$$
F(x y)=\int_{0}^{x} \int_{0}^{y} f(x y) d x d y
$$

dove $f(x y)$ è una funzione integrabile, nel senso del Lebesgue, in un certo campo $A$, contenente l'origine (o, o). Il Lebesgue ha dimostrato (ed è molto facile il vederlo) che, quasi dappertutto in $A$, esiste la derivata parziale $\frac{\partial F}{\partial x}$, uguale a $\int_{0}^{y} f(x y) d y . \mathrm{Ha}$ poi aggiunto ${ }^{\prime}$ ) che, trascurando un insieme di misura nulla, esiste ancbe la derivata seconda mista $\left(\frac{\partial^{2} F}{\partial x \partial y}\right)$, uguale a $f(x y)$. Questa derivata l'ho scritta racchiusa in una parentesi per ricordare che essa non è una vera derivata seconda. Per definizione, detto $\bar{A}$ l'insieme, di misura uguale a quella di $A$, che resta togliendo l'insieme che si è detto di trascurare, la $\left(\frac{\partial^{2} F}{\partial x \partial y}\right)$, in un punto $P(x, y)$ di $\bar{A}$, è il limite del rapporto

$$
\frac{\frac{\partial F\left(x, y^{\prime}\right)}{\partial x}-\frac{\partial F(x, y)}{\partial x}}{y^{\prime}-y},
$$

per $y^{\prime} \rightarrow y$, considerato soltanto nei punti $P^{\prime}\left(x, y^{\prime}\right)$ di $\bar{A}$. Ora io pongo la seguente questione: si puo dimostrare che, quasi dappertutto in $A$, esiste la derivata seconda mista $\frac{\partial^{2} F}{\partial x \partial y}$ [uguale necessariamente, per quanto sopra si è detto, a $\left.f(x y)\right]$ ? Si tratta cioè di

I) H. Lebesgue, Sur l'intégration des fonctions discontinues [Annales scientifiques de l'École Normale Supérieure (Paris), III ${ }^{e}$ série t. XXVII (19ro), pp. 36r-4jo], vedi anche Ch. J. DE LA VALléE Poussin, Cours d'Analyse, $2^{e}$ édition, t. II (Paris, Gauthier-Villars, I9I I), p. 122. 
togliere la restrizione, relativa a $y^{\prime}$, della definizione di $\left(\frac{\partial^{2} F}{\partial x \partial y}\right)$, e di dimostrare che quasi dappertutto esiste il limite di (I) per $y^{\prime}$ tendente liberamente a $y$.

Mi è riuscito di rispondere affermativamente alla domanda posta nel caso della $f(x y)$ limitata ed anche in alcuni casi notevoli in cui la $f(x y)$ è illimitata; ma non nel caso generale. Crede Ella che si possa rispondere affermativamente anche nel caso generale? Poichè Ella si è già occupata, e con tanto successo, di questioni prossime a questa, cosi mi permetto di importunarla con questa mia.

Ecco, frattanto, come si trattano il caso della $f(x y)$ limitata e quegli altri cui sopra ho accennato. Sia $\varphi(x y)$ una funzione integrabile linearmente rispetto alla $x$, in un certo intervallo $(a, b)$, per tutti $\mathrm{i}$ valori di $y$ di un intervallo $(c, d)$. Fissato un valore di $y$ di $(c, d)$, la $\varphi(x, y)$ è quasi dappertutto in $(a, b)$ la derivata di $\int_{a}^{x} \varphi(x y) d x$. Dico che si può determinare un insieme I di $(a, b)$, di misura $m(I)=b-a$, sul quale la $y(x, y)$ sia la derivata di $\int_{a}^{x} \varphi(x y) d x$, per tutti gli $y d i(c, d)$. Consideriamo $\mathrm{i}$ valori razionali di $y$, compresi in $(c, d)$. Ad ognuno di essi ccrrisponde un insieme $I_{y}$ di $(a, b)$, di misura $b-a$, sul quale la $\varphi$ è la derivata di $\int_{a}^{x} \varphi(x, y) d x$. Indichiamo con $I$ l'insieme dei punti comuni a tutti gli $I_{y}$ detti. $I$ ha misura uguale a $b-a$ e su esso la $\varphi(x, y)$ è la derivata di $\int_{a}^{x} \varphi(x, y) d x$ per tutti $\mathrm{i}$ valori razionali di $y$, compresi in $(c, d)$. Siano ora $\bar{y}$ un valore irrazionale $\mathrm{di}(c, d)$ e $y_{r}$ un valore razionale tendente $a \bar{y}$, per $r \rightarrow \infty$, e supponiamo [ipotesi $\alpha)$ ] che $\varphi\left(x y_{r}\right)$, per $r \rightarrow \infty$, tenda uniformemente a $\varphi(x \bar{y})$ su tutto $(a, b)$. Allora è $\varphi(x \bar{y})=\varphi\left(x, y_{r}\right)+\varepsilon\left(x, y_{r}\right)$, con $\varepsilon\left(x, y_{r}\right)$ tendente uniformemente a zero per $y_{r} \rightarrow \bar{y}$, e quindi

$$
\int_{a}^{x} \phi(x \bar{y}) d x=\int_{a}^{x} \phi\left(x, y_{r}\right) d x+\int_{a}^{x} \varepsilon\left(x, y_{r}\right) d x .
$$

Se $x_{1}$ è un punto qualunque di $I$, si ha perciò

$$
\begin{aligned}
\Lambda \int_{a}^{x_{1}} \varphi(x, \bar{y}) d x & =\varphi\left(x_{1}, y_{r}\right)+\bar{\varepsilon}, \\
\lambda \int_{a}^{x_{1}} \varphi(x \bar{y}) d x & =\varphi\left(x_{1}, y_{r}\right)+\overline{\bar{\varepsilon}},
\end{aligned}
$$

con $\bar{\varepsilon} \nrightarrow 0, \bar{\varepsilon} \nrightarrow 0$, per $y_{r} \rightarrow \bar{y}$; e analogamente per $\Lambda^{\prime} \int_{a}^{x_{t}} \varphi(x, \bar{y}) d x$ e $\lambda^{\prime} \int_{a}^{x_{s}} \varphi(x, \bar{y}) d x$. In $x$, esiste dunque la derivata di $\int_{a}^{x} \varphi(x \bar{y}) d x$ e tale derivata è uguale a

$$
\lim _{y \rightarrow-\bar{y}} \varphi\left(x_{1}, y_{r}\right)=\varphi\left(x_{1}, \bar{y}\right) \text {. }
$$


L'ipotesi $\alpha$ ) è soddisfatta se la $p(x, y)$ ha, rispetto ad $y$, la prima derivata parziale limitata, ed anche se è $\int_{a}^{b}\left|\frac{\partial \varphi}{\partial y}\right|^{1+\alpha} d x<M$, quaiunque sia l'y di $(c, d)$, con $\alpha>0$. Basta porre $\varphi(x y)=\int_{0}^{y} f(x, y) d y$, per avere i risultati accennati.

\section{LEONIDA TONELLI.}

II.

(da una lettera di Guibo Fubini a LEonida Tonelit).

\section{Egregio Professore,}

Ho pensato alla Sua questione senza leggere le Sue considerazioni, per non essere spinto a trattare il problema in un modo anzichè nell'altro. $\mathrm{E}$ mi sono accorto poi di aver battuto proprio la Sua strada, con piccole modificazioni, cosi da ottenere precisamente il risultato da Lei desiderato.

Conservo le Sue notazioni e pongo

$$
F(x y)=\int_{0}^{x} \int_{0}^{y} f(x y) d x d y
$$

Poichè, per l'ipotesi da Lei fatta, la $f(x y)$ è integrabile, secondo Lebesgue, in un campo superficiale, anche $|f(x y)|$ è integrabile. Cioè $f(x y)$ è la differenza di due funzioni positive integrabili. Io posso studiare ciascuna di queste due funzioni separatamente, cioè posso supporre $f(x y)$ positiva. Si dimostra col Suo metodo che vale la

$$
\frac{\partial F}{\partial x}=\int_{0}^{y} f(x y) d y
$$

per tutti i valori razionali di $y$ e per tutti $\mathrm{i}$ valori di $x$ che non appartengono a un certo gruppo $G$ di misura lineare nulla.

Sia $x$ un punto non appartenente a $G$; sia $y$ irrazionale; siano $y_{1}, y_{2}$ numeri razionali qualsiasi tali che $y_{1}<y<y_{2}$. Suppongo, per es., le $b, x, y, y_{1}, y_{2}$, positive. Allora 'è

$$
\int_{x}^{x+b} \int_{0}^{y_{1}} f d x d y \leq \int_{x}^{x+\hbar} \int_{0}^{y} f d x d y \leq \int_{x}^{x+\hbar} \int_{0}^{y_{2}} f d x d y .
$$

E ció perchè $f \supseteqq o$ e perchè il campo a cui è esteso il primo integrale è contenuto nel campo cui è esteso il secondo, il quale è contenuto in quello cui è esteso il terzo. Dividendo per $b$ e passando al limite per $b=0$, ne deduciamo: I numeri derivati a destra di $\int_{0}^{x} \int_{0}^{y} f d x d y$ sono compresi tra le derivate $d i \int_{0}^{x} \int_{0}^{y_{1}} f d x d y \mathrm{e}$ 
$\int_{0}^{x} \int_{0}^{y_{2}} f d x d y$, che per ipotesi esistono e valgano

$$
\int_{0}^{y_{1}} f d y \text { e } \int_{0}^{y_{2}} f d y \text {. }
$$

E cio qualunque siano $i$ numeri razionali $y_{1}$ e $y_{2}$ tali che $y_{1}<y<y_{2}$. Passando al limite per $y_{1}=y_{2}=y$, poichè $\int_{0}^{y} f d x$ è funzione non decrescente e continua della $y$, si trova che la derivata di $F(x, y)$ rispetto ad $x$, qualunque sia $y$, e purchè $x$ non appartenga a $G$, vale proprio

$$
\int_{0}^{y} f d y
$$

che noi possiamo ora derivare rispetto ad $y$, etc. etc.

Guido Fubini. 